\title{
Energy Savings Achievable in Connection Preserving Energy Saving Algorithms
}

\author{
Seh Chun $\mathrm{Ng}^{* \dagger}$ \\ * School of Electrical and \\ Information Engineering \\ University of Sydney \\ $\dagger$ National ICT Australia Limited \\ Sydney, Australia \\ Email: sehchun@ee.usyd.edu.au
}

\author{
Guoqiang $\mathrm{Mao}^{* \dagger}$ \\ ${ }^{*}$ School of Electrical and \\ Information Engineering \\ University of Sydney \\ ${ }^{\dagger}$ National ICT Australia Limited \\ Sydney, Australia \\ Email: guoqiang@ee.usyd.edu.au
}

\author{
Brian D.O. Anderson ${ }^{\ddagger \S}$ \\ ${ }^{\ddagger}$ Research School of Information \\ Sciences and Engineering \\ Australian National University \\ $\S$ National ICT Australia Limited \\ Canberra, Australia \\ Email: brian.anderson@nicta.com.au
}

\begin{abstract}
Energy saving is an important design consideration in wireless sensor networks. In this paper, we analyze the energy savings that can be achieved in a sensor network where each sensor is capable of reducing its transmission power from a maximum power $p_{m}$, compared with that in a sensor network where each sensor can only transmit at a constant power level $p_{m}$. To achieve a fair comparison, we assume sensors in both types of sensor networks are connected to the same set of neighbors, i.e. no connection is lost as a result of a sensor reducing its transmission power. We further assume that sensors are distributed in a given area following a Poisson distribution with known node density and the radio propagation is described by a log-normal model. Ignoring boundary effect, we establish analytically the probability for a sensor to achieve an energy saving of at least $h$ dB. We also obtain the expected percentage of energy savings which can be substantial. The research reported in the paper helps to answer questions such as whether the energy savings achieved by using a sensor with a variable-transmission-power (and the consequent extension of its lifetime) justify the additional cost involved in manufacturing it.
\end{abstract}

Index Terms - sensor network, log-normal shadowing, transmit power, energy savings

\section{INTRODUCTION}

Wireless sensor networks have been widely used for monitoring environments and detecting events. A wireless sensor network consists of a set of sensors equipped with sensing hardware, and able to communicate with each other via radio links. Sensors are normally battery-operated. In wireless sensor networks, each sensor plays two important roles. It sends data it has collected and it acts as a relay to other sensors. Therefore, failure of some sensors due to exhaustion of battery power will cause topology change in a network, and in the worst case, the network will be disconnected prematurely. Since failed sensors are normally hard to replace, energy saving becomes an important network design consideration in wireless sensor networks. One of the common goals in designing wireless sensor networks is to make sure sensing and communication tasks can be carried out and in the mean time the network lifetime is maximized.

National ICT Australia is funded by the Australian Government Department of Communications, Information Technology and the Arts and the Australian Research Council through Backing Australia's Ability and the ICT Centre of Excellence Program.
The approaches taken so far by various researchers on improving energy efficiency have been very diverse [1]. The studies on energy saving mechanisms cover almost all aspects of network design, including choices of hardware. There are also many energy savings mechanisms that are carried out in higher level aspects of network operation. These include, but are not limited to, routing protocols, scheduling mechanisms, and adjustment of transmission power. In [2], the authors survey the energy-aware routing techniques which are suitable for wireless sensor networks. These routing protocols use different approaches to meet the energy constraint in wireless sensor networks. For example, redundant data are aggregated to reduce the number of transmissions. There is also an approach that chooses different paths at different times to transmit data, instead of using a single optimal path, to balance energy consumption across all sensors. In [3], the authors review 15 scheduling mechanisms in wireless sensor networks. These scheduling mechanisms achieve the objective of minimizing energy consumption by scheduling sensors into work/sleep cycles.

In this paper, we study the possible energy savings achieved through adjustment of transmission power. Deciding a transmission power level is a complicated matter. It not only affects the amount of energy consumed, but also determines the establishment of direct links to the nearby nodes, and hence affects the network topology and the paths that can be used for routing to the destination. In this paper, we investigate the possible energy savings that can be achieved in a sensor network where sensors are allowed to reduce their transmission power without losing any connections to their neighbor nodes, via some connection preserving algorithms. More specifically, we analyze the energy savings that can be achieved in a sensor network where each sensor is capable of reducing its transmission power from a maximum power $p_{m}$, compared with that in a sensor network where each sensor can only transmit at a constant power level $p_{m}$. To achieve a fair comparison, we assume sensors in both types of sensor networks are connected to the same set of neighbors, i.e. no connection is lost as a result of a sensor reducing its transmission power. Reducing transmission power may have other complicated effects on the 
network. For example, on the one hand, it reduces interference to the transmission of other sensor pairs which is beneficial; on the other hand, it decreases SNR which consequently increases packet error rate. We do not consider these effects in the paper. We further assume that sensors are distributed in a given area following a Poisson distribution with known node density and the radio propagation is described by a log-normal model. Ignoring boundary effect, we establish analytically the probability for a sensor to achieve an energy saving of at least $h \mathrm{~dB}$. We also obtain the expected percentage of energy savings. The research reported in the paper helps to answer questions such as whether the energy savings achieved by using a sensor with a variable-transmission-power (and the consequent extension of its lifetime) justify the additional cost involved in manufacturing it.

The rest of this paper is organized as follows: Section II introduces related work on energy savings. Section III defines the system model considered in this paper, followed by section IV which presents the basic theoretical analysis and derivations. Section V presents further analysis and numerical data, which isolates key aspects of the average percentage of energy savings obtained from section IV. Finally, section VI concludes this paper and discusses possible future work.

\section{RELATED WORK}

Energy saving related topics have attracted many studies over the years. Research in the area has been done from different perspectives [1]. Among them, the widely adopted way of achieving energy saving is via minimizing the transmission power. Deng et al. [4] identify the optimal transmission range that maximizes the ratio of per-hop transmission distance to the energy consumption. In their paper, nodes are assumed to be Poissonly distributed over a given region. Ignoring boundary effect, the authors identify the average distance progress for each hop, which is defined to be the difference between the before-hop distance (distance between current node and the destination node) and after-hop distance (distance between next node in the path and the destination node). With taking into account the energy required to both transmit and receive packets, the authors find the optimal transmission range that maximizes the ratio of distance progress to energy consumption.

In [5], Clementi et al. study the problem of minimizing the transmission power while meeting the requirement that any pair of nodes in the network is at most $k$ hops away. In the paper, a finite set of nodes are allowed to transmit using different transmission ranges. A node is directly connected to another node if their Euclidean distance is less than the transmission range. A determinstic path loss model in an ideal environment (path loss exponent $=2$ ) has been used to relate the transmission range to the transmission power. The authors analyze the problem of finding the best set of transmission ranges where the sum of the transmission power of all nodes is minimum, at the same time ensuring that any two nodes in the network are at most $k$ hops away.
In [6], the performance of different dynamic transmissionpower-control algorithms are reported. These algorithms show that dynamic transmission-power control will reduce power consumption. The studies are different from our work in a several aspects: a) The algorithms may increase or decrease the transmission power to meet some predefined bound for the number of neighbors of each node; b) In some of the studies, the establishment of links between two nodes is decided by the packet reception rate; c) Simulation results are reported without analytic backing. Indeed, to the best of our knowledge, there is no analytical result obtained on the possible energy savings in the scenario mentioned in section I.

In this paper, we assume that wireless nodes are uniformly, independently, and identically distributed over a twodimensional area. This type of node distribution has been commonly used in many major studies of some fundamental network properties, such as connectivity [7], [8] and capacity [9], [10]. However, instead of following the analysis of these papers based on a simplistic unit disk channel model, we consider channel model with certain randomness. Consideration of channel randomness is necessary because the non-negligible impacts of channel randomness, like log-normal shadowing and Rayleigh fading, to the network connectivity have been reported in the recent work [11], [12], [13]. In this paper, the log-normal model has been selected to carry out the analysis. However, the work in this paper can be readily extended to other types of stochastic channel models.

\section{SYSTEM MODEL}

In this paper we consider that wireless sensors are randomly, independently and identically distributed over a twodimensional area according to a Poisson point process. The node density is known to be $\lambda$. Let us denote by $X_{i}$ the position of node $i$. Then we denote by $R_{i j}=\left\|X_{i}-X_{j}\right\|$ the Euclidean distance between a pair of randomly chosen nodes $i$ and $j$. Since $R_{i j}$ are also independently and identically distributed random variables for different pairs of $(i, j)$, we drop the index $i j$ in the rest of the paper where the indices are not important.

Node $i$ can directly communicate with node $j$ if the received power at node $j$, denoted by $P_{r}$, is above a certain known threshold $p_{t h}$. The relationship between $P_{r}$ and the Euclidean distance between nodes $i$ and $j, R$, follows the log-normal model [14],

$$
P_{r}=p_{0}-10 \alpha \log _{10} \frac{R}{d_{0}}+G
$$

where $P_{r}$ is the received power at node $j, p_{0}$ is the power at a reference distance $d_{0}, \alpha$ is the path loss exponent, $G$ is a Gaussian distributed random variable with zero mean and variance $\sigma^{2}$. The random variable $G$ reflects the shadowing effect in the environment. Both $P_{r}$ and $p_{0}$ are in $\mathrm{dBm}$ unit.

Initially, node $i$ transmits at the maximum transmission power $p_{m}$, which results in a reference power $p_{0}$ at a reference distance $d_{0}$. This leads to the establishment of a set of direct connections between node $i$ and some other 
nodes, i.e. its neighbors. After that, node $i$ is allowed to reduce its transmission power continuously while maintaining connections with the same set of neighbors. The assumption that node $i$ can adjust its power continuously is mainly used to simplify the later analysis, which does not critically rely on the assumption. Given the distribution function of energy savings, which will be derived later, the results can be easily modified for nodes having a set of discrete transmission power levels. The difference between the maximum transmission power $p_{m}$ and the minimum transmission power required for maintaining connections with the same set of neighbors as those when node $i$ transmits at the maximum power $p_{m}$, is in fact the energy that could be saved by node $i$ without any sacrifice in connections. The energy savings that can be achieved for a randomly chosen node is of course a random variable depending both on the distribution of distances between the node and its neighbors and on the randomness in the log-normal model. Denote this random variable by $H$; in the next section we shall derive the distribution of $H$.

\section{ANALYSIS}

Select an arbitrary node, say node $i$. Let the maximum transmission power be $p_{m}$ such that it results in a received power of $p_{0}$ (in the absence of shadowing effect) at reference distance $d_{0}$. Let $r_{0}$ be a sufficiently large positive value such that the probability that a node, whose Euclidean distance to node $i$ is larger than $r_{0}$, is connected to node $i$ can be ignored. So, we shall only consider nodes whose Euclidean distance to node $i$ is within $r_{0}$.

Denote by $R$ the random variable representing the Euclidean distance between a randomly chosen node $j \neq i$ within the circular area $A$ centered at node $i$ and with a radius $r_{0}$, and node $i$. Assuming node $j$ is at a known and fixed distance $r<$ $r_{0}$ to node $i$, i.e. $R=r$, the received power $P_{r}$ at node $j$ from node $i$ is Gaussian distributed with mean $p_{0}-10 \alpha \log _{10} \frac{r}{d_{0}}$ and variance $\sigma^{2}$. Therefore, the conditional probability that node $j$ receives a power from node $i$, which transmits at the maximum power $p_{m}$, higher than the threshold power level $p_{t h}$, i.e. node $j$ is connected to node $i$, given their Euclidean distance equals to $r$, is

$$
\begin{aligned}
& \operatorname{Pr}\left\{P_{r} \geq p_{t h} \mid R=r\right\} \\
& =\int_{p_{t h}}^{\infty} \frac{1}{\sqrt{2 \pi} \sigma} \exp \left(-\frac{\left(P_{r}-p_{0}+10 \alpha \log _{10} \frac{r}{d_{0}}\right)^{2}}{2 \sigma^{2}}\right) \mathrm{d} P_{r} \\
& =\int_{p_{t h}-p_{0}+10 \alpha \log _{10} \frac{r}{d_{0}}}^{\infty} \frac{1}{\sqrt{2 \pi} \sigma} \exp \left(-\frac{x^{2}}{2 \sigma^{2}}\right) \mathrm{d} x
\end{aligned}
$$

Note that when deriving this equation, we have made an assumption that the power received by a randomly chosen node when node $i$ is transmitting is independent of the power received by another randomly chosen node when node $i$ is transmitting. Equivalently, the random path losses are assumed to be independent. These assumptions are also used later.

Since nodes are assumed to be Poissonly distributed, and node $j$ is within the circular area $A$ centered at node $i$ and with radius $r_{0}$, the probability density function of $R$ is $f_{R}(r)=$ $\frac{2 r}{r_{0}^{2}}$, for $0 \leq r \leq r_{0}$. Combining this with Eq. (2) gives the probability that an arbitrary node within $A$ is connected to node $i$. That is,

$$
\begin{aligned}
\operatorname{Pr} & \left\{P_{r} \geq p_{t h}\right\} \\
= & \int_{0}^{r_{0}} \operatorname{Pr}\left\{P_{r} \geq p_{t h} \mid R=r\right\} \times \frac{2 r}{r_{0}^{2}} \mathrm{~d} r \\
= & \int_{0}^{r_{0}}\left(\int_{p_{t h}-p_{0}+10 \alpha \log _{10} \frac{r}{d_{0}}}^{\infty} \frac{1}{\sqrt{2 \pi} \sigma} \exp \left(-\frac{x^{2}}{2 \sigma^{2}}\right) \mathrm{d} x\right) \\
& \times \frac{2 r}{r_{0}^{2}} \mathrm{~d} r
\end{aligned}
$$

Note that we can further simplify Eq. (3) by changing the order of double integral. Firstly, the original sample space:

$$
\left\{\begin{array}{l}
r \in\left[0, r_{0}\right] \\
x \in\left[p_{t h}-p_{0}+10 \alpha \log _{10} \frac{r}{d_{0}}, \infty\right)
\end{array}\right.
$$

is equivalent to a new sample space, consists of two areas $S_{1}$ and $S_{2}$ (as shown in Fig. 1):

$$
\begin{aligned}
& S_{1}=\left\{\begin{array}{l}
r \in\left[0, d_{0} 10^{\frac{x-p_{t h}+p_{0}}{10 \alpha}}\right] \\
x \in\left(-\infty, p_{t h}-p_{0}+10 \alpha \log _{10} \frac{r_{0}}{d_{0}}\right]
\end{array}\right. \\
& S_{2}=\left\{\begin{array}{l}
r \in\left[0, r_{0}\right] \\
x \in\left[p_{t h}-p_{0}+10 \alpha \log _{10} \frac{r_{0}}{d_{0}}, \infty\right)
\end{array}\right.
\end{aligned}
$$

Then, we can change Eq. (3) to:

$$
\begin{aligned}
\operatorname{Pr} & \left\{P_{r} \geq p_{t h}\right\} \\
= & \int_{0}^{r_{0}} \int_{p_{t h}-p_{0}+10 \alpha \log _{10} \frac{r}{d_{0}}}^{\infty} \frac{1}{\sqrt{2 \pi} \sigma} \exp \left(-\frac{x^{2}}{2 \sigma^{2}}\right) \mathrm{d} x \\
& \times \frac{2 r}{r_{0}^{2}} \mathrm{~d} r \\
= & \int_{-\infty}^{p_{t h}-p_{0}+10 \alpha \log _{10} \frac{r_{0}}{d_{0}}}\left(\int_{0}^{d_{0} 10^{\frac{x-p_{t h}+p_{0}}{10 \alpha}}} \frac{2 r}{r_{0}^{2}} \mathrm{~d} r\right) \\
& \times \frac{1}{\sqrt{2 \pi} \sigma} \exp \left(-\frac{x^{2}}{2 \sigma^{2}}\right) \mathrm{d} x \\
& +\int_{p_{t h}-p_{0}+10 \alpha \log _{10} \frac{r_{0}}{d_{0}}}^{\infty}\left(\int_{0}^{r_{0}} \frac{2 r}{r_{0}^{2}} \mathrm{~d} r\right) \frac{1}{\sqrt{2 \pi} \sigma} \\
& \times \exp \left(-\frac{x^{2}}{2 \sigma^{2}}\right) \mathrm{d} x \\
= & \int_{-\infty}^{p_{t h}-p_{0}+10 \alpha \log _{10} \frac{r_{0}}{d_{0}}}\left(\frac{d_{0}^{2}}{r_{0}^{2}} 10^{\frac{x-p_{t h}+p_{0}}{5 \alpha}}\right) \frac{1}{\sqrt{2 \pi} \sigma} \\
& \times \exp \left(-\frac{x^{2}}{2 \sigma^{2}}\right) \mathrm{d} x \\
& +\int_{p_{t h}-p_{0}+10 \alpha \log _{10} \frac{r_{0}}{d_{0}}}^{\infty} \frac{1}{\sqrt{2 \pi} \sigma} \exp \left(-\frac{x^{2}}{2 \sigma^{2}}\right) \mathrm{d} x
\end{aligned}
$$

Similarly, the probability that an arbitrarily chosen node in $A$ is connected to node $i$ with received power at least $p_{t h}+h$ 


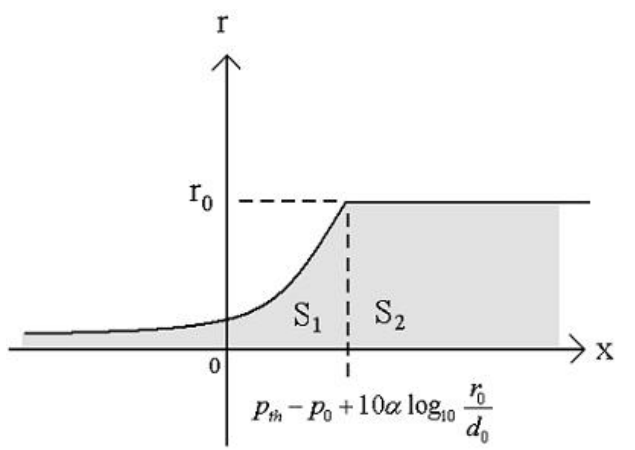

Fig. 1. An area graph displays the region of integration for Eq. (3).

will be

$$
\begin{aligned}
\operatorname{Pr} & \left\{P_{r} \geq p_{t h}+h\right\} \\
= & \int_{-\infty}^{p_{t h}+h-p_{0}+10 \alpha \log _{10} \frac{r_{0}}{d_{0}}}\left(\frac{d_{0}^{2}}{r_{0}^{2}} 10^{\frac{x-p_{t h}-h+p_{0}}{5 \alpha}}\right) \frac{1}{\sqrt{2 \pi} \sigma} \\
& \times \exp \left(-\frac{x^{2}}{2 \sigma^{2}}\right) \mathrm{d} x \\
& +\int_{p_{t h}+h-p_{0}+10 \alpha \log _{10} \frac{r_{0}}{d_{0}}}^{\infty} \frac{1}{\sqrt{2 \pi} \sigma} \exp \left(-\frac{x^{2}}{2 \sigma^{2}}\right) \mathrm{d} x
\end{aligned}
$$

Following from the previous results, we can derive the probability that an arbitrary connected node is connected to node $i$ with received power greater than $p_{t h}+h$ :

$$
\begin{aligned}
& \operatorname{Pr}\left\{P_{r} \geq p_{t h}+h \mid P_{r} \geq p_{t h}\right\} \\
& =\frac{\operatorname{Pr}\left\{P_{r} \geq p_{t h}+h\right\}}{\operatorname{Pr}\left\{P_{r} \geq p_{t h}\right\}}
\end{aligned}
$$

As $r_{0} \rightarrow \infty$, the above conditional probability becomes

$$
\begin{aligned}
& \operatorname{Pr}\left\{P_{r} \geq p_{t h}+h \mid P_{r} \geq p_{t h}\right\} \\
& =\frac{\int_{-\infty}^{\infty}\left(d_{0}^{2} 10^{\frac{x-p_{t h}-h+p_{0}}{5 \alpha}}\right) \frac{1}{\sqrt{2 \pi} \sigma} \exp \left(-\frac{x^{2}}{2 \sigma^{2}}\right) \mathrm{d} x}{\int_{-\infty}^{\infty}\left(d_{0}^{2} 10^{\frac{x-p_{t h}+p_{0}}{5 \alpha}}\right) \frac{1}{\sqrt{2 \pi} \sigma} \exp \left(-\frac{x^{2}}{2 \sigma^{2}}\right) \mathrm{d} x} \\
& =\frac{d_{0}^{2} 10^{\frac{-p_{t h}-h+p_{0}}{5 \alpha}} \exp \left(\frac{\sigma^{2} \log ^{2} 10}{50 \alpha^{2}}\right)}{d_{0}^{2} 10^{\frac{-p_{t h}+p_{0}}{5 \alpha}} \exp \left(\frac{\sigma^{2} \log ^{2} 10}{50 \alpha^{2}}\right)} \\
& =10^{\frac{-h}{5 \alpha}}
\end{aligned}
$$

where $\log ($.$) is natural logarithm.$

Clearly, if the arbitrarily chosen node is connected to node $i$ and has a received power greater than $p_{t h}+h$, node $i$ is able to reduce its transmission power by $h$ without losing the connection to this specific node.

Let $N$ be the number of nodes in $A$ that are connected to node $i$. From Mukherjee and Avidor's recent work [15], we know that $N$ is a Poissonly distributed random variable with mean $\mu=\lambda \pi r_{0}^{2} \times \operatorname{Pr}\left\{P_{r} \geq p_{t h}\right\}$, where $\lambda$ is the node density. This property relies on our previous assumption that power received at an arbitrary node is independent of power received at another arbitrary node. So the mean value as $r_{0} \rightarrow \infty$ agrees

$$
\mu=\lambda \pi d_{0}^{2} \exp \left(\frac{\sigma^{2} \log ^{2} 10}{50 \alpha^{2}}+\frac{\left(p_{0}-p_{t h}\right) \log 10}{5 \alpha}\right)
$$

Given the probability distribution function of random variable $N$ and the equations derived so far, the probability that all neighbors of node $i$, are connected with received power greater than $p_{t h}+h$, can finally be derived. This is the probability distribution of random variable $H$ defined in section III. Among all the possible values for $N$, a special consideration should be taken for $N=0$. That is the case when node $i$ is an isolated node (no neighbor nodes). We assume no energy saving can be achieved by node $i$ if it is an isolated node.

$$
\begin{aligned}
& \operatorname{Pr}\{H \geq h\} \\
& =\sum_{n=1}^{\infty} \frac{\mu^{n} \exp (-\mu)}{n !}\left(\operatorname{Pr}\left\{P_{r} \geq p_{t h}+h \mid P_{r} \geq p_{t h}\right\}\right)^{n} \\
& =\sum_{n=1}^{\infty} \frac{\left(\mu \times 10^{\frac{-h}{5 \alpha}}\right)^{n} \exp (-\mu)}{n !} \\
& =\exp (-\mu) \times\left[\exp \left(\mu 10^{\frac{-h}{5 \alpha}}\right)-1\right]
\end{aligned}
$$

In the above equation, we consider that if a node has no neighbor (isolated node), it cannot deliver any energy savings at all. Therefore in the above equation $n$ starts from 1 , instead of 0 . The probability density function of $H$ is then

$$
\begin{aligned}
f_{H}(h) & =\frac{d}{d h} \operatorname{Pr}\{H \leq h\} \\
& =\frac{\log 10}{5 \alpha} 10^{\frac{-h}{5 \alpha}} \mu \exp (-\mu) \exp \left(\mu 10^{\frac{-h}{5 \alpha}}\right)
\end{aligned}
$$

From the probability density function of $H$, we can also obtain the average percentage of energy saving for a randomly selected node $i$. Define $p_{n}=p_{m}-h$. That is, $p_{n}$ is the transmission power achievable by node $i$ where the connections to all its neighbors are maintained. Denote by $p_{m}^{*}$ and $p_{n}^{*}$ the corresponding $p_{m}$ and $p_{n}$ in milliwatts instead of $\mathrm{dBm}$. Denote by $h^{*}$ the corresponding $h$ in decimal units. Their relationship can be explained by the following equation:

$$
p_{n}^{*}=\frac{p_{m}^{*}}{h^{*}}
$$

Then, we can derive the fraction of energy savings $g(h)$ as

$$
g(h)=\frac{p_{m}^{*}-p_{n}^{*}}{p_{m}^{*}}=1-\frac{1}{h^{*}}=1-10^{-\frac{h}{10}}
$$

Using Eq. (6), the expected fraction of energy savings is,

$$
\begin{aligned}
E[g(h)]= & \int_{0}^{\infty}\left(1-10^{-\frac{h}{10}}\right) \frac{\log 10}{5 \alpha} 10^{\frac{-h}{5 \alpha}} \mu \exp (-\mu) \\
& \times \exp \left(\mu 10^{\frac{-h}{5 \alpha}}\right) \mathrm{d} h \\
= & -\int_{1}^{0}\left(1-y^{\frac{\alpha}{2}}\right) \mu \exp (-\mu) \exp (\mu y) \mathrm{d} y \\
= & 1-\exp (-\mu)-(-\mu)^{-\frac{\alpha}{2}} \exp (-\mu) \\
& \times\left[\Gamma\left(\frac{\alpha}{2}+1,-\mu\right)-\Gamma\left(\frac{\alpha}{2}+1\right)\right]
\end{aligned}
$$


where $\mu$ is as in Eq. (4), $\Gamma($.$) is the Gamma function defined$ as

$$
\Gamma(a)=\int_{0}^{\infty} t^{a-1} \exp (-t) \mathrm{d} t
$$

and $\Gamma(a, z)$ is the upper incomplete Gamma function defined as

$$
\Gamma(a, z)=\int_{z}^{\infty} t^{a-1} \exp (-t) \mathrm{d} t
$$

Eq. (7) indicate that the expected energy savings achievable by using a sensor with variable transmission power is determined by the path loss exponent and the average node degree.

\section{NUMERICAL EVALUATION}

In this section, we evaluate the impact of various parameters, i.e. average node degree $\mu$, variance of shadowing $\sigma^{2}$ and path loss exponent $\alpha$, on the expected percentage of energy savings. The variance of shadowing $\sigma^{2}$ exerts its impact on expected percentage of energy savings by affecting the average node degree $\mu$.

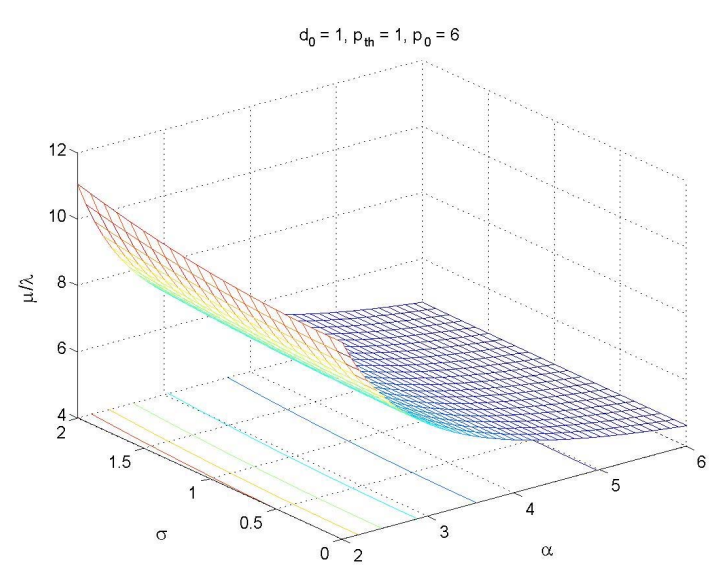

Fig. 2. Impact of path loss exponent $\alpha$ and standard deviation of shadowing $\sigma$ on average number of neighbors $\mu$ per node density $\lambda$.

Eq. (7) shows that the expected percentage of energy savings depends only on the average number of neighbors $\mu$ (also known as the average node degree) and the path loss exponent $\alpha$. However, from Eq. (4) we know that the average node degree is not only directly proportional to the node density $\lambda$, but also related to two environment parameters: the path loss exponent $\alpha$, and the shadowing variance $\sigma^{2}$. Fig. 2 shows the impact of these environment parameters on the average node degree. Since the average node degree $\mu$ is known to be directly proportional to the node density $\lambda$, we choose to plot the ratio $\mu / \lambda$ as $\mathrm{z}$-axis to remove the impact of $\lambda$ from the plot. The result shows that shadowing has a positive impact on the average node degree, i.e. a larger shadowing variance leads to a higher average node degree, whereas a larger path loss exponent has a negative impact on it. The changes in the average node degree due to different $\sigma$ are small, compared with the significant impact from $\alpha$, especially for lower values of $\alpha$.

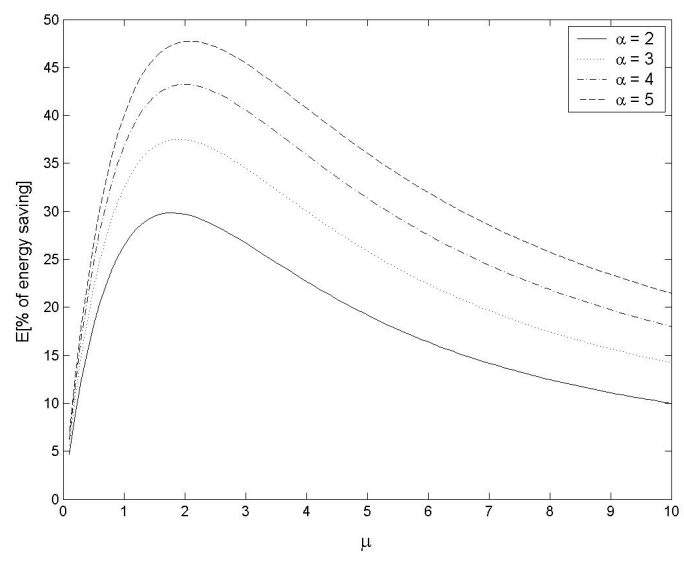

Fig. 3. Expected percentage of energy savings with different $\mu$, where $\alpha$ between 2 to 5 . Reference [14] suggests that $\alpha=2$ represents free space environment, $\alpha \in[2,3]$ represents different indoor environment, and $\alpha \in$ $[3,5]$ represents shadowed urban area.

Considering the previous analysis in Eq. (7), it is obvious that different values of $\alpha$ will significantly affect the expected percentage of energy savings of a wireless sensor. Different values of $\alpha$ represents different environments that the wireless sensors are deployed. Reference [14] suggests that normally $\alpha=2$ is used to represent a free space environment, $\alpha \in$ $[2,3]$ represents different indoor environment, and $\alpha \in[3,5]$ represents shadowed urban area. Fig. 3 demonstrates the expected percentage of energy savings for different values of $\alpha$ corresponding to these environments. It shows that sensors in a larger $\alpha$ value environment generally experience higher expected percentages of energy savings. This suggests that higher energy savings are to be expected in the obstructed environment.

Fig. 3 shows the expected percentage of energy savings increases with $\mu$ when the values of $\mu$ is small; it peaks at a certain value of $\mu$ and the value of $\mu$ that maximizes the expected percentage of energy savings varies slightly with $\alpha$; finally the expected percentage of energy savings drops with $\mu$ when the value of $\mu$ is large. The expected percentage of energy savings decreases with $\mu$ when the value of $\mu$ is large because it is harder to gain energy savings when each sensor gets connected to a large number of neighbors. On the other hand, the expected percentage of energy savings also drops at a very small value of node degree. This phenomenon is mainly caused by the increase in probability that a node will become isolated in the network. Such probability is given by $\operatorname{Pr}\{$ a node is isolated $\}=\exp (-\mu)$. Therefore, a decrease in the average node degree will result in an increase in probability of a node becoming isolated. An isolated node will not yield any energy saving.

Another interesting result from Fig. 3 is that there is always an optimal point for each curve in the plot. That is, in each environment which is characterized by the value of the path loss exponent $\alpha$, there is an optimal average node degree $\mu$ that leads to the maximum energy savings. Fig. 4 and Fig. 5 


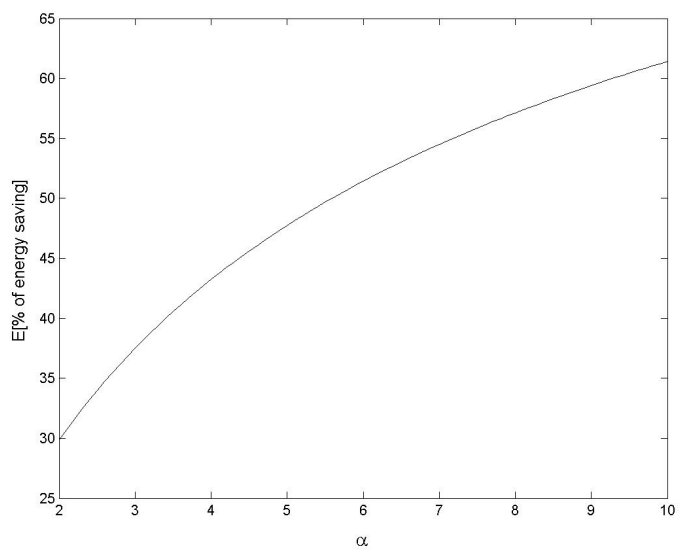

Fig. 4. Maximum expected percentage of energy savings (i.e. the optimal point of each line in Fig. 3) with $\alpha \in[2,10]$.

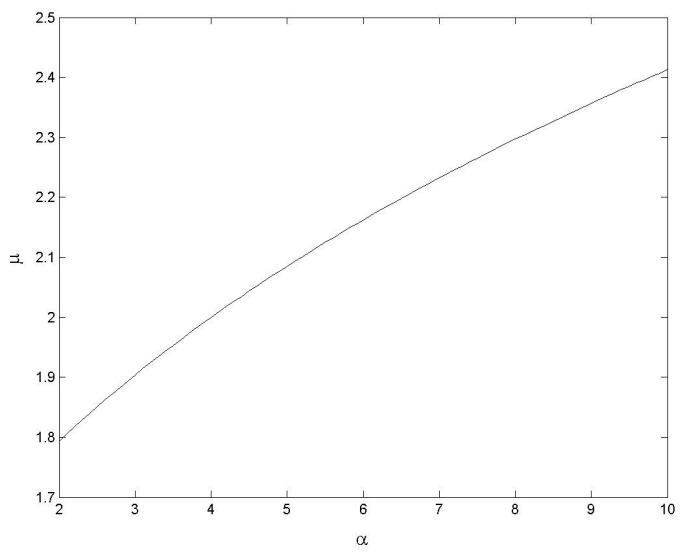

Fig. 5. The corresponding average node degree where the maximum expected percentage of energy savings can be obtained, for $\alpha \in[2,10]$.

show the maximum expected percentage of energy savings that can be achieved for each value of $\alpha$, and the combination of $\alpha$ and $\mu$ that lead to the maximum energy savings respectively. Fig. 4 shows that on average, at least $30 \%$ of energy savings is achievable for sensors with variable-transmission-power at most environmental conditions. This result shows that sensors with variable-transmission-power will significantly outperform sensors with constant-transmission-power in terms of energy efficiency and sensor lifetime. Fig. 5 shows that the range of average node degree to achieve the maximum expected percentage of energy savings is narrow (from 1.8 to 2.41). Hence, keeping the average node degree approximately at 2 will gain near to optimal performance on energy savings.

\section{CONCLUSION AND FUTURE WORK}

In this paper, we have studied the achievable energy savings in a sensor network where the sensors are capable of reducing their transmission power while maintaining the connections to their neighbors. The wireless channel is modeled by the log-normal model. In general, the sensors with variable- transmission-power will gain significant energy savings, especially in an environment with a high path loss exponent.

The results obtained in this study are of practical value. Firstly, the analytical results show that sensors with variabletransmission-power can deliver significant energy savings, which possibly justifies their use in a real network. Secondly, the results also suggest that a near-to-optimal average energy saving is obtainable by a sensor network if the average node degree is kept approximately to 2 .

Our existing work analyzes the achievable energy savings under the condition that no connection is lost as a result of reducing transmission power. That means that the network topology is maintained during the course of reducing transmission power. However, this does not necessarily need to be the case. In the future, we will extend current work to examine the achievable energy savings where the reduction of transmission power is allowed to change the network topology, as long as the network still preserves some network properties, such as connectivity or $k$-connectivity.

\section{REFERENCES}

[1] A. Ephremides, "Energy concerns in wireless networks," IEEE Wireless Communications, vol. 9, no. 4, pp. 48-59, August 2002.

[2] J. N. Al-Karaki and A. E. Kamal, "Routing techniques in wireless sensor networks: a survey," IEEE Wireless Communications, vol. 11, no. 6, pp. 6-28, December 2004.

[3] L. Wang and Y. Xiao, "A survey of energy-efficient scheduling mechanisms in sensor networks," Mobile Networks and Applications, vol. 11, no. 5, pp. 723-740, October 2006.

[4] J. Deng, Y. S. Han, P.-N. Chen, and P. K. Varshney, "Optimal transmission range for wireless ad hoc networks based on energy efficiency," IEEE Transactions on Communications, vol. 55, no. 9, September 2007.

[5] A. E. F. Clementi, P. Penna, and R. Silvestri, "On the power assignment problem in radio networks," Mobile Networks and Applications, vol. 9, no. 2, pp. 125-140, April 2004.

[6] J. Jeong, D. Culler, and J.-H. Oh, "Empirical analysis of transmission power control algorithms for wireless sensor networks," University of California at Berkeley, Tech. Rep. UCB/EECS-2005-16, November 2005.

[7] M. Desai and D. Manjunath, "On the connectivity in finite ad hoc networks," IEEE Communications Letters, vol. 6, no. 10, pp. 437-439, October 2002

[8] P. Gupta and P. R. Kumar, "Critical power for asymptotic connectivity in wireless networks," Stochastic Analysis, Control, Optimization and Applications: A Volume in Honor of W.H. Fleming, W.M. McEneaney, G. Yin, and Q. Zhang (Eds.), pp. 547-566, 1998.

[9] —, "The capacity of wireless networks," IEEE Transactions on Information Theory, vol. 46, no. 2, pp. 388-404, March 2000.

[10] M. Grossglauser and D. N. C. Tse, "Mobility increases the capacity of ad hoc wireless networks," IEEE/ACM Transactions on Networking, vol. 10, no. 4, pp. 477-486, August 2002.

[11] C. Bettstetter and C. Hartmann, "Connectivity of wireless multihop networks in a shadow fading environment," Wireless Networks, vol. 11, no. 5, pp. 571-579, 2005.

[12] D. Miorandi and E. Altman, "Connectivity in one-dimensional ad hoc networks: a queueing theoretical approach," Wireless Networks, vol. 12, no. 5 , pp. 573-587, 2006.

[13] D. Miorandi, E. Altman, and G. Alfano, "The impact of channel randomness on coverage and connectivity of ad hoc and sensor networks," IEEE Transactions on Wireless Communications, vol. 7, no. 3, pp. 1062-1072, March 2008.

[14] T. S. Rappaport, Wireless Communications: Principles and Practice, 2nd ed. Prentice Hall PTR, December 2002.

[15] S. Mukherjee and D. Avidor, "Connectivity and transmit-energy considerations between any pair of nodes in a wireless ad hoc network subject to fading," IEEE Transactions on Vehicular Technology, vol. 57, no. 2, pp. 1226-1242, March 2008. 